\title{
Exact solutions of transaction cost nonlinear models for illiq- uid markets
}

Javed Hussain

Department of Mathematics, Sukkur IBA University, airport road Sukkur, Sindh, Pakistan.

\begin{abstract}
The aim of this study is to show that the Reduced Differential Transform Algorithm (RDTA) can be applied to highly nonlinear evolution equations appearing in quantitative finance. In particular, we compute exact solutions of nonlinear PDEs arising by relaxing the transaction-cost assumption in the illiquid Black-Scholes market. Moreover, we also aim to study the impact of the absence and presence of price slippage impact in the illiquid Black-Scholes model with transaction-cost.
\end{abstract}

Keywords: Option pricing, relaxed Black-Scholes assumptions, evolution equation, differential transform.

2020 MSC: 35Q91, 91G80.

(C)2021 All rights reserved.

\section{Introduction}

The classical assumptions of the Black-Scholes market are randomness of stocks, the constancy of interest rate and volatility of stocks, no transaction on trading stocks, and liquidity of market (i.e., trading can be done at any time, in any unit of fractions). Under these assumptions, the euro-type option prices satisfy the following linear differential equations,

$$
\frac{\partial u}{\partial t}+\frac{\sigma^{2} s^{2}}{2} \frac{\partial^{2} u}{\partial s^{2}}+r s \frac{\partial u}{\partial s}-r u=0,
$$

where $u$ is the value of option, $\sigma$ is the volatility of stocks, $r$ is the interest rate. For the investors who invest with large investments and especially for the incomplete markets (i.e., where not every derivative cannot be priced) these Black-Scholes assumptions give rise to unrealistic nonlinear models. In such cases the volatility can be taken as time $t$-dependent, stock price $s$, and even on the premium $u$ of the option and its Greeks.

There have been several attempts to solve and analyze (1.1) analytically and numerically in abstract and concrete spaces, see for example, Jódar [24]; Rodrigo [31]; Ponsoda [11]; Ankudinova [2]; Bohner [5]; Cen [9], and Allahviranloo [1]. On assuming the market with friction and non-competitive and with transaction cost, we will reach an illiquid/rigid market and in such a market, the prices of the derivative will evolve non linearly. Bakstein [3] studied liquidity as a blend of transaction cost by the trader and the

Email address: javed.brohi@iba-suk.edu.pk (Javed Hussain)

doi: $10.22436 /$ jmcs.023.03.08 
impact of price slippage. We aim to study such nonlinear equations involving transaction cost, feed-back effect, and with \& without price slippage impact.

The large trading effects in the illiquid markets have been studied by a number of authors. For instance, Frey and Stremme in [19], and Frey and Patie in [18] studied these effects on the price and concluded that the following choice of volatility should be made,

$$
\widehat{\sigma}\left(t, s, \frac{\partial u}{\partial s}, \frac{\partial^{2} u}{\partial s^{2}}\right)=\frac{\sigma}{1-\rho s \lambda(s) \frac{\partial^{2} u}{\partial s^{2}}},
$$

where $\sigma$ is the standard volatility, $\rho \in \mathbb{R}$ is a measure of the market liquidity, and $\lambda$ is the market price of risk quantifying the liquidity profile of the market. In order to solve the nonlinear Black-Scholes equation with volatility (1.2), Chmakova in [6] took $\lambda$ as a constant. In [17, 19], a generalized version of Black-Scholes pricing PDE was derived of the form,

$$
\frac{\partial u}{\partial t}+\frac{\sigma^{2} s^{2}}{2\left(1-\lambda(s) \frac{\partial^{2} u}{\partial s^{2}}\right)^{2}} \frac{\partial^{2} u}{\partial s^{2}}+r s \frac{\partial u}{\partial s}-r V=0, \quad u(s, T)=h(s), \text { where } s>0 .
$$

The case $h$ is assumed to be Lipchitz, the well-posedness of the solution have been shown in [28].

On treating the market price $\lambda$ as $\lambda(s) \equiv 1$, recall the approximation $\frac{1}{(1-x)^{2}} \approx 1+2 x+O\left(x^{3}\right)$, when $x$ is sufficiently small. Therefore by assuming that $\left|\rho s \frac{\partial^{2} u}{\partial s^{2}}\right|$ is sufficiently small, and using the mentioned approximation we can rewrite the equation (1.3) as following. Financially assuming $\rho s \frac{\partial^{2} u}{\partial s^{2}}$ to be sufficiently small corresponds to the case of low impact of hedging, see for instance A4 in [19],

$$
\frac{\partial u}{\partial t}+\frac{s^{2}}{2}\left(\sigma \sqrt{\left(1+2 \rho s \frac{\partial^{2} u}{\partial s^{2}}\right)}\right)^{2} \frac{\partial^{2} u}{\partial s^{2}}=0, \quad u(s, T)=h(s), \text { where } s>0 .
$$

An alternate, semi-martingale based derivation of the above problem has been given in [16]. By feedback effect in a transaction cost model means that the volatility $\sigma \sqrt{1+2 \rho S u_{s s}}$ depends on the sign of Greek"Gamma" $u_{s s}$. Moreover, following continuous-time feedback effects equation for illiquid markets were modeled by Bakstein and Howison in [3],

$$
\frac{\partial u}{\partial t}+\frac{\sigma^{2} s^{2}}{2} \frac{\partial^{2} u}{\partial s^{2}}\left(1+2 \rho s \frac{\partial^{2} u}{\partial s^{2}}\right)+\frac{\rho^{2}(1-\alpha) \sigma^{2} s^{4}}{2}\left(\frac{\partial^{2} u}{\partial s^{2}}\right)^{3}+r s \frac{\partial u}{\partial s}-r u=0
$$

subject to initial condition $u(s, 0)=f(s)$, where $f$ is the payoff derivative, $\rho \in \mathbb{R}-\{0\}$ is the measure of the market liquidity, and $\alpha$ is the measure of the impact of price slippage impact, due to trade, experienced by participants of market participants. The case when $\alpha=1$ corresponds to the no slippage condition and equation (1.5) reduces to the following model,

$$
\frac{\partial u}{\partial t}+\frac{\sigma^{2} s^{2}}{2} \frac{\partial^{2} u}{\partial s^{2}}\left(1+2 \rho s \frac{\partial^{2} u}{\partial s^{2}}\right)+r s \frac{\partial u}{\partial s}-r u=0
$$

The fundamental objective is to study the evolution equations (1.4), (1.5), and (1.6) subject to some concrete initial conditions using reduced differential transform (RDT) algorithm. The equations (1.1), (1.3), (1.4), (1.6), and (1.5) are also closely related with several models appearing in natural sciences, for this we refer to $[26,27,33]$. Moreover, we refer to some recent closely related work to studies done in this paper, see $[8,21-23,30,32]$.

\section{Brief description of reduced differential transform algorithm}

This section is aimed to introduce the reduced differential transform algorithm to solve the nonlinear evolution equation. We will define RDTA and list some of its basic definitions and important properties. 
2.1. Definition and properties of differential transform

Let us start by defining the 1-D reduced differential transform of a smooth (i.e., $\mathrm{C}^{\infty}(\mathbb{R})$ function.

Definition 2.1 ([25]). Suppose function $u(t, x)$ be $C^{\infty}(\mathbb{R})$-function and is analytic, then the differential transform of $u(t, x)$ is followin,

$$
u_{k}(x)=\left.\frac{1}{k !} \frac{\partial^{k} u}{\partial t^{k}}(t, x)\right|_{t=0}, \quad k=0,1,2,3, \ldots,
$$

where $u_{k}(x)$ can be treated as the the $t$-dimensional spectrum transformed function.

The differential inverse transform of $u_{k}(x)$ is defined as follows:

$$
u(t, x)=\sum_{k=0}^{\infty} u_{k}(x) t^{k}=\sum_{k=0}^{\infty}\left(\left.\frac{1}{k !} \frac{\partial^{k} u}{\partial t^{k}}(t, x)\right|_{t=0}\right) t^{k} .
$$

Based on the above we have the following theorem listing the basic properties of reduced differential transform.

Theorem 2.2 ([25]). For any smooth functions $u, v$ the reduce differential transform of $u$ and $v$ satisfies following properties.

(i) Linearity: for any linear combination of $u$ and $v$, i.e., $w(t, x)=a u(t, x)+b v(t, x)$, where $a, b \in \mathbb{R}$, the reduced differential transform is

$$
W_{k}(x)=a_{k}(x)+V_{k}(x), \quad k \in \mathbb{N},
$$

where $u_{k}, v_{k}$, and $w_{k}$ are differential transforms of $u, v$, and $w$, respectively.

(ii) If $u(t, x)=x^{m} t^{n}$, then reduce differential transform of $u$ is $u_{k}(x)=x^{m} \delta(k-n), k \in \mathbb{N}$.

(iii) If $v(x, t)=x^{m^{n}} t^{n}(x, t)$, then reduce differential transform of $v$ is $v_{k}(x)=x^{m} u_{k-n}(x), k \in \mathbb{N}$.

(iv) If $w(x, t)=\mathfrak{u}(x, t) v(x, t)$, then reduce differential transform of $w$ is,

$$
W_{k}(x)=\sum_{r=0}^{k} V_{r}(x) u_{k-r}(x), k \in \mathbb{N}
$$

(v) If $v(x, t)=\frac{\partial^{r}}{\partial t^{r}} u(x, t)$, then reduce differential transform of $v$ is,

$$
v_{k}(x)=\frac{(k+r) !}{k !} u_{k+r}(x), k \in \mathbb{N} .
$$

(vi) Space derivative of $u$ is invariant under differential transform, more precisely, if $v(t, x)=\frac{\partial u}{\partial x}(x, t)$, then reduce differential transform of $v$ is $v_{k}(x)=\frac{\partial u_{k}}{\partial x}(x)$.

2.2. Applications of differential transform to nonlinear evolution equations: a generic algorithm

Consider the following nonlinear evolution equation,

$$
u_{t}(t, x)=A u(t, x)+B u(t, x)+f(t, u(t, x)), \quad u(0, x)=h(x),
$$

where $A$ is linear operator, $B$ is nonlinear linear operator and $f$ is some linear smooth function of $x$. Suppose that variables can be separated, i.e., $u(t, x)$ can be written as product of functions of $x$ and $t$, i.e., $u(t, x)=f(x) g(t)$, where $f(x)$ and $g(t)$ smooth functions of space and time variables, respectively. Based on the properties of one dimensional differential transform, the function $u(t, x)$ can be represented as follows:

$$
u(t, x)=\left(\sum_{i=0}^{\infty} F(i) x^{i}\right)\left(\sum_{i=0}^{\infty} G(j) t^{j}\right)=\sum_{k=0}^{\infty} u_{k}(x) t^{k},
$$


where $u_{k}(x)$ is called $t$-dimensional spectrum function of $u(t, x)$. Using Definition 2.1 and suitable properties from Theorem 2.2 we may take differential transform of problem (2.1) we get the following algorithm consisting of recursive set of equations,

$$
(k+1) u_{k+1}(x)=A u_{k}(x)+B u_{k}(x)+F\left(u_{k}(x)\right), \quad k=1,2,3, \ldots, \quad u_{0}(x)=h(x),
$$

where $A u_{k}(x), B u_{k}(x)$, and $F\left(u_{k}(x)\right)$ are the transformations of the functions $A u(t, x), B u(t, x)$, and $f(t, x)$, respectively. Using the above relation one can compute the $\mathfrak{u}_{k}$ from $u_{k-1}$, for all $k=1,2,3, \ldots$ and get sequence of smooth function $\left(u_{k}(x)\right)_{k=0}^{\infty}$. Then the series solution of the evolution equation (2.1) can be recovered from the following inverse differential transform,

$$
\widetilde{u}_{n}(t, x)=\sum_{k=0}^{n} u_{k}(x) t^{k}
$$

Hence, taking limit of partial sums will lead to solution of evolution equation,

$$
u(t, x)=\lim _{n \rightarrow \infty} \widetilde{u}_{n}(t, x)=\sum_{k=0}^{\infty} u_{k}(x) t^{k}
$$

\subsection{Convergence of RDT algorithm}

We now present some interesting recent results from [29] about the convergence of RDT algorithm.

Theorem 2.3. The solution series $\sum_{k=0}^{\infty} u_{k}(x) t^{k}$, described in (2.2), converges, if there exists $\gamma \in(0,1)$ such that $\left\|u_{k+1}(x) t^{k+1}\right\| \leqslant \gamma\left\|u_{k}(x) t^{k}\right\|$, for all $k \in \mathbb{N} \cup\{0\}$. Moreover, if $\sum_{k=0}^{\infty} u_{k}(x) t^{k}$ converges to $u(t, x)$, then error between the truncated sum $\sum_{k=0}^{n} \mathfrak{u}_{k}(x) t^{k}$ and $\mathrm{u}(\mathrm{t}, \mathrm{x})$ can be controlled by following inequality,

$$
\left\|u(t, x)-\sum_{k=0}^{n} u_{k}(x) t^{k}\right\| \leqslant \frac{\gamma^{n+1}}{\gamma-1}\left\|u_{0}\right\|
$$

\section{Pricing European option with transaction cost and price slippage impact}

In this section, we aim to deal with the continuous-time feedback effects equation for illiquid markets developed by Bakstein and Howison in [3]. Assume that market trades two kinds of assets, namely, illiquid risky (say stock) asset and liquid risk-free (say bond or money market account). The model that describes the evolution of the European option satisfies the following initial value problem (see Theorem 3.1 in $[3,14])$,

$$
\frac{\partial u}{\partial t}+\frac{1}{2} \sigma^{2} s^{2} \frac{\partial^{2} u}{\partial s^{2}}\left(1+2 \rho s \frac{\partial^{2} u}{\partial s^{2}}\right)+\frac{1}{2} \rho^{2}(1-\alpha)^{2} \sigma^{2} s^{4}\left(\frac{\partial^{2} u}{\partial s^{2}}\right)^{3}+r s \frac{\partial u}{\partial s}-r u=0,
$$

subject to initial condition

$$
\left.u(s, 0)=\frac{1}{\rho}\left[\left(\frac{-1 \pm \sqrt{1-(1-\alpha)^{2}}}{(1-\alpha)^{2}}\left(\ln \left(\frac{s}{k}\right)+\frac{s_{0}}{k}\right) s\right)-s_{0}\right]\right] .
$$

Here $t$ denotes time, $s$ is the current price of the stock, $\rho \geqslant 0$ is the measure of the market liquidity, $\sigma$ is volatility, $u(s, t)$ is the option price, and $\alpha$ is the measure of the impact of price slippage on all participants of the market. Using Definition 2.1 and properties of differential transform from Theorem 2.2 to equation (3.1), we arrive at the following recursive Algorithm,

$$
(k+1) u_{k+1}=-\left[\frac{\sigma^{2} s^{2}}{2} \frac{\partial^{2} u_{k}}{\partial s^{2}}+\rho \sigma^{2} s^{3} A_{k}+\frac{\rho^{2}(1-\alpha)^{2} \sigma^{2} s^{4}}{2} B_{k}+r s \frac{\partial u_{k}}{\partial s}-r u_{k}\right],
$$


where

$$
A_{k}=\sum_{h=0}^{k} \frac{\partial^{2} u}{\partial s^{2}}(s, k) \frac{\partial^{2} u}{\partial s^{2}}(s, k-h), \quad B_{k}=\sum_{k=0}^{h} \sum_{m=0}^{k} \frac{\partial^{2} u(s, m)}{\partial s^{2}} \frac{\partial^{2} u(s, h-m)}{\partial s^{2}} \frac{\partial^{2} u(s, h-k)}{\partial s^{2}} .
$$

Here $u_{k}(x)$ is the transformed function. $A_{k}$ and $B_{k}$ are the transformed functions for nonlinear terms.

Let us move towards computing the solution of the initial value problem by computing the $u_{k}$, for all $k=0,1,2, \ldots$, from recurrence relation (3.3).

When $k=0$ : From (3.3), we have,

$$
u_{1}=-\left[\frac{\sigma^{2} s^{2}}{2} \frac{\partial^{2} u_{0}}{\partial s^{2}}+\rho \sigma^{2} s^{3} A_{0}+\frac{\rho^{2}(1-\alpha)^{2} \sigma^{2} s^{4}}{2} B_{0}+r s \frac{\partial u_{0}}{\partial s}-r u_{0}\right]
$$

Now using the initial condition, we get,

$$
\frac{\partial \mathrm{u}_{0}}{\partial \mathrm{s}}=\frac{1}{\rho}\left(\ln \left(\frac{\mathrm{s}}{\mathrm{k}}\right)+\frac{\mathrm{s}_{0}}{\mathrm{k}}+\frac{1}{\rho}\right)\left(\frac{-1 \pm \sqrt{1-(1-\alpha)^{2}}}{(1-\alpha)^{2}}\right), \quad \frac{\partial^{2} \mathrm{u}_{0}}{\partial s^{2}}=\frac{1}{s \rho}\left(\frac{-1 \pm \sqrt{1-(1-\alpha)^{2}}}{(1-\alpha)^{2}}\right) .
$$

Therefore,

$$
A_{0}=\left(\frac{\partial^{2} u_{0}}{\partial s^{2}}\right)^{2}=\left(\frac{1}{s \rho} \frac{-1 \pm \sqrt{1-(1-\alpha)^{2}}}{(1-\alpha)^{2}}\right)^{2}, \quad B_{0}=\left(\frac{\partial^{2} u_{0}}{\partial s^{2}}\right)^{3}=\left(\frac{1}{s \rho} \frac{-1 \pm \sqrt{1-(1-\alpha)^{2}}}{(1-\alpha)^{2}}\right)^{3}
$$

Substituting the values of $u_{0}, \frac{\partial u_{0}}{\partial s}, \frac{\partial^{2} u_{0}}{\partial s^{2}}, A_{0}$, and $B_{0}$ in (3.4),

$$
\begin{aligned}
u_{1}= & -\frac{\sigma^{2} s^{2}}{2}\left(\frac{1}{s \rho} \frac{-1 \pm \sqrt{1-(1-\alpha)^{2}}}{(1-\alpha)^{2}}\right)-\rho \sigma^{2} s^{3}\left(\frac{1}{s \rho} \frac{-1 \pm \sqrt{1-(1-\alpha)^{2}}}{(1-\alpha)^{2}}\right)^{2} \\
& -\frac{\rho^{2}(1-\alpha)^{2} \sigma^{2} s^{4}}{2}\left(\frac{1-1 \pm \sqrt{1-(1-\alpha)^{2}}}{s \rho}\right)^{3}-\frac{r s}{\rho}\left(\ln \left(\frac{s}{k}\right)+\frac{s_{0}}{k}+\frac{1}{\rho}\right)\left(\frac{-1 \pm \sqrt{1-(1-\alpha)^{2}}}{(1-\alpha)^{2}}\right) \\
& +r\left(\frac{1}{\rho} \frac{-1 \pm \sqrt{1-(1-\alpha)^{2}}}{(1-\alpha)^{2}}\left\{\ln \left(\frac{s}{k}\right)+\frac{s_{0}}{k}\right\} s-s_{0}\right) .
\end{aligned}
$$

After simplifications we get,

$$
u_{1}=-\frac{1}{\rho}\left[\left(\frac{-1 \pm \sqrt{1-(1-\alpha)^{2}}}{(1-\alpha)^{2}}\right) r s+r s_{0}\right]
$$

Now the case when $k=1$ : Using $k=1$ in equation (3.4), we get

$$
2 u_{2}=-\left[\frac{\sigma^{2} s^{2}}{2} \frac{\partial^{2} u_{1}}{\partial s^{2}}+\rho \sigma^{2} s^{3} A_{1}+\frac{\rho^{2}(1-\alpha)^{2} \sigma^{2} s^{4}}{2} B_{1}+r s \frac{\partial u_{1}}{\partial s}-r u_{1}\right] .
$$

Using (3.5) it follows that,

$$
\frac{\partial u_{1}}{\partial s}=-\frac{r}{\rho} \frac{-1 \pm \sqrt{1-(1-\alpha)^{2}}}{(1-\alpha)^{2}}, \quad \frac{\partial^{2} u_{1}}{\partial s^{2}}=0,
$$

and therefore,

$$
A_{1}=2\left(\frac{\partial^{2} u_{0}}{\partial s^{2}}\right)\left(\frac{\partial^{2} u_{1}}{\partial s^{2}}\right)=0, \quad B_{1}=2\left(\frac{\partial^{2} u_{0}}{\partial s^{2}}\right)^{2}\left(\frac{\partial^{2} u_{1}}{\partial s^{2}}\right)=0
$$


Substituting the values of $u_{1}, \frac{\partial u_{1}}{\partial s}, \frac{\partial^{2} u_{1}}{\partial s^{2}}, A_{1}$, and $A_{2}$ in equation (3.6) we get,

$$
u_{2}=\frac{1}{2 \rho}\left[\frac{-1 \pm \sqrt{1-(1-\alpha)^{2}}}{(1-\alpha)^{2}}\{r(r s-r s)\}-r^{2} s_{0}\right]=-\frac{r^{2} s_{0}}{2 \rho}
$$

When $k=2$ : By putting $k=2$, into the equation (3.3) we get

$$
3 u_{3}=-\left[\frac{\sigma^{2} s^{2}}{2} \frac{\partial^{2} u_{2}}{\partial s^{2}}+\rho \sigma^{2} s^{3} A_{2}+\frac{\rho^{2}(1-\alpha)^{2} \sigma^{2} s^{4}}{2} B_{2}+r s \frac{\partial u_{2}}{\partial s}-r u_{2}\right]
$$

By computing $A_{2}$ and $B_{2}$ we get,

$$
A_{2}=\frac{\partial^{2} u_{0}}{\partial s^{2}} \frac{\partial^{2} u_{2}}{\partial s^{2}}+\frac{\partial^{2} u_{1}}{\partial s^{2}} \frac{\partial^{2} u_{1}}{\partial s^{2}}+\frac{\partial^{2} u_{2}}{\partial s^{2}} \frac{\partial^{2} u_{0}}{\partial s^{2}}=0, \quad B_{2}=2\left(\frac{\partial^{2} u_{0}}{\partial s^{2}}\right)^{2}\left(\frac{\partial^{2} u_{2}}{\partial s^{2}}\right)+\left(\frac{\partial^{2} u_{1}}{\partial s^{2}}\right)^{2} \frac{\partial^{2} u_{0}}{\partial s^{2}}=0 .
$$

Hence we get,

$$
u_{3}=-\frac{r^{3}}{6} \frac{1}{\rho} s_{0}
$$

Next, we claim inductively that,

$$
A_{k}=B_{k}=0, \text { for all } k \geqslant 2,
$$

and

$$
u_{k}=-\frac{r^{k}}{k !} \frac{s_{0}}{\rho}
$$

Suppose

$$
A_{i}=B_{i}=0, \text { for all } i \in[2, k-1] \cap \mathbb{N},
$$

and

$$
\frac{\partial u_{i}}{\partial s}=0 \text { and } \frac{\partial^{2} u_{i}}{\partial s^{2}}=0, \text { for all } i \in[2, k-1] \cap \mathbb{N}, \quad u_{i}=-\frac{r^{i}}{i !} \frac{s_{0}}{\rho} \text {, for all } i \in[2, k-1] \cap \mathbb{N} \text {. }
$$

Therefore,

$$
\begin{aligned}
k u_{k} & =-\left[\frac{\sigma^{2} s^{2}}{2} \frac{\partial^{2} u_{k-1}}{\partial s^{2}}+\rho \sigma^{2} s^{3} A_{k-1}+\frac{1}{2} \rho^{2}(1-\alpha)^{2} \sigma^{2} s^{4} B_{k-1}+r s \frac{\partial u_{k-1}}{\partial s}-r u_{k-1}\right]=-r\left(\frac{r^{k-1}}{(k-1) !} \frac{s_{0}}{\rho}\right), \\
u_{k} & =-\frac{r^{k}}{k !} \frac{s_{0}}{\rho}, \text { for all } k \geqslant 2 .
\end{aligned}
$$

Finally, we are in a position to write the solution of initial value problem (3.1)-(3.2), which can be given as,

$$
u(s, t)=u_{0}(s)+\sum_{k=1}^{\infty} u_{k}(s) t^{k}=\frac{1}{\rho}\left[\left(\frac{-1 \pm \sqrt{1-(1-\alpha)^{2}}}{(1-\alpha)^{2}}\left\{\ln \left(\frac{s}{k}\right)+\frac{s_{0}}{k}\right\} s\right)-s_{0}\right]-\frac{s_{0}}{\rho} \sum_{k=2}^{\infty} \frac{(r t)^{k}}{k !} .
$$

Thus the exact solution of the problem is rapidly obtained as follows

$$
\left.\mathrm{u}(\mathrm{s}, \mathrm{t})=\frac{1}{\rho}\left[\left(\frac{-1 \pm \sqrt{1-(1-\alpha)^{2}}}{(1-\alpha)^{2}}\left(\left\{\ln \left(\frac{s}{\mathrm{k}}\right)+\frac{s_{0}}{\mathrm{k}}\right\}-r \mathrm{t}\right\} s\right)\right)-s_{0} \mathrm{e}^{\mathrm{rt}}\right] .
$$

The above solution obtained through the reduced differential transform algorithm is in a complete agreement with traveling waves solution obtained by Esekon in [14] (Theorem 3.2). The plot of the solution, for $(s, t) \in[0,10] \times[0,10]$, and taking $\rho=0.01, s_{0}=4, k=10$, and $r=0.06$, is given in Figure 1 . 


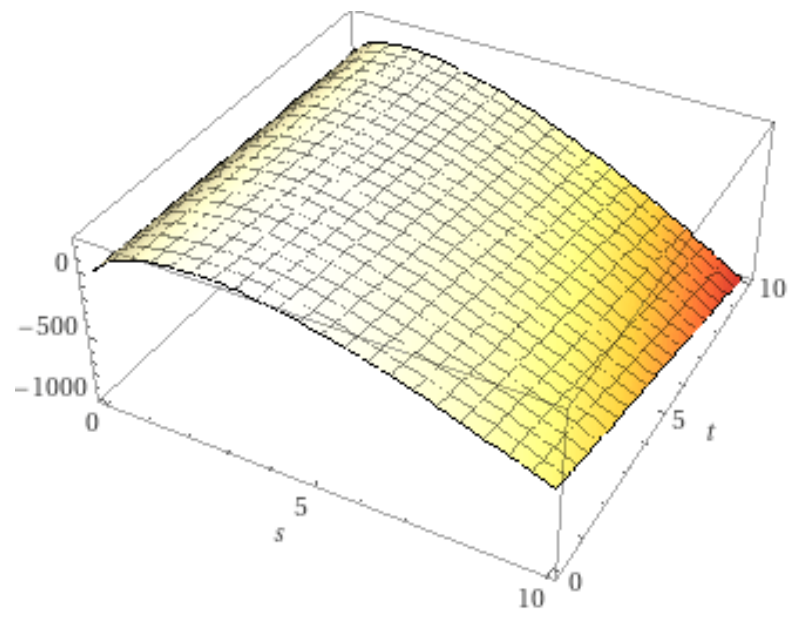

Figure 1

\section{Pricing European option with transaction cost and no slippage impact}

In this section, our aim is to study the nonlinear Black-Scholes evolution equations arising as a result of incorporating the transaction and hence illiquidity of the market, in the absence of impact of price slippage impact. For further details of the model, we refer to [13] (Theorem 3.0.2). The model leading the evolution of the price of European option with transaction cost and no slippage leads to the following:

$$
\frac{\partial u}{\partial t}+\frac{\sigma^{2} s^{2}}{2} \frac{\partial^{2} u}{\partial s^{2}}\left(1+2 \rho s \frac{\partial^{2} u}{\partial s^{2}}\right)+r s \frac{\partial u}{\partial s}-r u=0,
$$

subject to initial condition

$$
u(s, 0)=s-\frac{\sqrt{s s_{0}}}{\rho}-\frac{s_{0}}{4 \rho} .
$$

Here $s$ is the running price of the stock, $\rho$ is a measure of the market liquidity, $\sigma$ is volatility, $u(s, t)$ is the option price, and $\alpha$ is the measure of the impact of the price slippage experienced by all market participants.

Using Definition 2.1 and properties of differential transform from Theorem 2.2 to equation (4.1), we arrive at the following recursive algorithm,

$$
(k+1) u_{k+1}=-\left[\frac{\sigma^{2} s^{2}}{2} \frac{\partial^{2} u_{k}}{\partial s^{2}}+\rho \sigma^{2} s^{3} A_{k}+r s \frac{\partial u_{k}}{\partial s}-r u_{k}\right],
$$

where $u_{k}$ is the transformed function and $A_{k}=\sum_{k=0}^{h} \frac{\partial^{2} u(s, k)}{\partial s^{2}} \cdot \frac{\partial^{2} u(s, h-k)}{\partial s^{2}}$, is the transformed function for nonlinear terms.

When $k=0$ : Using $k=0$ in equation (4.3), we get

$$
u_{1}=-\left[\frac{\sigma^{2} s^{2}}{2} \frac{\partial^{2} u_{0}}{\partial s^{2}}+\rho \sigma^{2} s^{3} A_{0}+r s \frac{\partial u_{0}}{\partial s}-r u_{0}\right]
$$

From the above initial condition (4.2), we compute the first and the second partial derivatives of (4.2) and $A_{0}$ and then we have,

$$
\frac{\partial u_{0}}{\partial s}=1-\frac{1}{2 \rho} \sqrt{\frac{s_{0}}{s}}, \quad \frac{\partial^{2} u_{0}}{\partial s^{2}}=\frac{1}{4 s \rho} \sqrt{\frac{s_{0}}{s}}, \quad A_{0}=\left(\frac{\partial^{2} u_{0}}{\partial s^{2}}\right)^{2}=\left(\frac{1}{4 s \rho} \sqrt{\frac{s_{0}}{s}}\right)^{2} .
$$


Substituting $\frac{\partial u_{0}}{\partial s}, \frac{\partial^{2} u_{0}}{\partial s^{2}}$, and $A_{0}$ in equation (4.4), we get

$$
\begin{aligned}
u_{1} & =-\frac{\sigma^{2} s^{2}}{2}\left(\frac{1}{4 s \rho} \sqrt{\frac{s_{0}}{s}}\right)+\rho \sigma^{2} s^{3}\left(\frac{1}{4 s \rho} \sqrt{\frac{s_{0}}{s}}\right)^{2}+r s\left(1-\frac{1}{2 \rho} \sqrt{\frac{s_{0}}{s}}\right)-r\left(s-\frac{\sqrt{s s_{0}}}{\rho}-\frac{s_{0}}{4 \rho}\right) \\
& =-\frac{\sqrt{s s_{0}}}{\rho}\left(\frac{\sigma^{2}}{8}+\frac{r}{2}\right)-\frac{s_{0}}{4 \rho}\left(\frac{1}{4} \sigma^{2}+r\right) .
\end{aligned}
$$

When $k=1$ : Using $k=1$ in equation (4.3), we get

$$
2 u_{2}=-\left[\frac{\sigma^{2} s^{2}}{2} \frac{\partial^{2} u_{1}}{\partial s^{2}}+\rho \sigma^{2} s^{3} A_{1}+r s \frac{\partial u_{1}}{\partial s}-r u_{1}\right] .
$$

Now let us compute the first and the second partial derivative of (4.5) with respect to $s$ to obtain the following,

$$
\frac{\partial u_{1}}{\partial s}=-\frac{1}{2 \rho} \sqrt{\frac{s_{0}}{s}}\left(\frac{\sigma^{2}}{8}+\frac{r}{2}\right), \quad \frac{\partial^{2} u_{1}}{\partial s^{2}}=\frac{1}{4 s \rho} \sqrt{\frac{s_{0}}{s}}\left(\frac{\sigma^{2}}{8}+\frac{r}{2}\right),
$$

and

$$
A_{1}=2 \frac{\partial^{2} u_{0}}{\partial s^{2}} \frac{\partial^{2} u_{1}}{\partial s^{2}}=\frac{s_{0}}{8 s^{3} \rho^{2}}\left(\frac{\sigma^{2}}{8}+\frac{r}{2}\right) .
$$

By substituting the values of $\frac{\partial u_{1}}{\partial s}, \frac{\partial^{2} u_{1}}{\partial s^{2}}$, and of $A_{1}$ in equation (4.6), we get

$$
\begin{aligned}
2 u_{2}= & -\frac{\sigma^{2} s^{2}}{2}\left(-\frac{1}{2 \rho} \sqrt{\frac{s_{0}}{s}}\left(\frac{\sigma^{2}}{8}+\frac{r}{2}\right)\right)-\rho \sigma^{2} s^{3}\left(\frac{s_{0}}{8 s^{3} \rho^{2}}\left(\frac{\sigma^{2}}{8}+\frac{r}{2}\right)\right)-r s\left(-\frac{1}{2 \rho} \sqrt{\frac{s_{0}}{s}}\left(\frac{\sigma^{2}}{8}+\frac{r}{2}\right)\right) \\
& +\frac{r \sqrt{s s_{0}}}{\rho}\left(\frac{\sigma^{2}}{8}+\frac{r}{2}\right)+\frac{r s_{0}}{4 \rho}\left(\frac{\sigma^{2}}{4}+r\right), \\
= & -\frac{\sqrt{s s_{0}}}{2 \rho}\left(\frac{\sigma^{2}}{8}+\frac{r}{2}\right)^{2}-\frac{s_{0}}{8 \rho}\left(\frac{\sigma^{2}}{4}+r\right)^{2} .
\end{aligned}
$$

When $k=2$ : For $k=2$ the equation (4.3) becomes,

$$
3 u_{3}=-\left[\frac{\sigma^{2} s^{2}}{2} \frac{\partial^{2} u_{2}}{\partial s^{2}}+\rho \sigma^{2} s^{3} A_{2}+r s \frac{\partial u_{2}}{\partial s}-r u_{2}\right]
$$

Now using the equation (4.7) to compute,

$$
\frac{\partial u_{2}}{\partial s}=-\frac{1}{4 \rho} \sqrt{\frac{s_{0}}{s}}\left(\frac{\sigma^{2}}{8}+\frac{r}{2}\right)^{2}, \quad \frac{\partial^{2} u_{2}}{\partial s^{2}}=\frac{1}{8 s \rho} \sqrt{\frac{s_{0}}{s}}\left(\frac{\sigma^{2}}{8}+\frac{r}{2}\right)^{2},
$$

and

$$
A_{2}=\frac{\partial^{2} u_{0}}{\partial s^{2}} \frac{\partial^{2} u_{2}}{\partial s^{2}}+\frac{\partial^{2} u_{1}}{\partial s^{2}} \frac{\partial^{2} u_{1}}{\partial s^{2}}+\frac{\partial^{2} u_{2}}{\partial s^{2}} \frac{\partial^{2} u_{0}}{\partial s^{2}}=\frac{s_{0}}{8 s^{3} \rho^{2}}\left(\frac{\sigma^{2}}{8}+\frac{r}{2}\right)^{2}
$$

By substituting the values of $\frac{\partial u_{2}}{\partial s}, \frac{\partial^{2} u_{2}}{\partial s^{2}}$, and $A_{2}$ in (4.8) we get,

$$
\begin{aligned}
3 u_{3}= & -\frac{\sigma^{2} s^{2}}{2}\left(\frac{1}{8 s \rho} \sqrt{\frac{s_{0}}{s}}\left(\frac{\sigma^{2}}{8}+\frac{r}{2}\right)^{2}\right)-\frac{\rho \sigma^{2} s^{3}}{8 s^{2} \rho^{2}} \frac{s_{0}}{s}\left(\frac{\sigma^{2}}{8}+\frac{r}{2}\right)^{2}+\frac{r s}{4 \rho} \sqrt{\frac{s_{0}}{s}}\left(\frac{\sigma^{2}}{8}+\frac{r}{2}\right)^{2} \\
& +\frac{r \sqrt{s s_{0}}}{2 \rho}\left(\frac{\sigma^{2}}{8}+\frac{r}{2}\right)^{2}+\frac{r}{8 \rho} s_{0}\left(\frac{\sigma^{2}}{4}+r\right)^{2} .
\end{aligned}
$$


After some simplification, we get

$$
u_{3}=-\frac{\sqrt{s s_{0}}}{6 \rho}\left(\frac{\sigma^{2}}{8}+\frac{r}{2}\right)^{3}-\frac{s_{0}}{24 \rho}\left(\frac{\sigma^{2}}{4}+r\right)^{3} .
$$

Inductively, it follows that

$$
u_{k}=-\frac{\sqrt{s s_{0}}}{k ! \rho}\left(\frac{\sigma^{2}}{8}+\frac{r}{2}\right)^{k}-\frac{s_{0}}{4 k ! \rho}\left(\frac{\sigma^{2}}{4}+r\right)^{k}, k \in \mathbb{N}
$$

Thus we are in a position to explicitly write the solution of (4.1)-(4.2). Using (4.2) and (4.9) in the inverse differential transformed, we get

$$
\begin{aligned}
u(s, t) & =u_{0}(x)+\sum_{k=1}^{\infty} u_{k}(s) t^{k} \\
& =s-\frac{\sqrt{s s_{0}}}{\rho}-\frac{s_{0}}{4 \rho}+\sum_{k=1}^{n}\left(-\frac{\sqrt{s s_{0}}}{k ! \rho}\left(\frac{\sigma^{2}}{8}+\frac{r}{2}\right)^{k}-\frac{s_{0}}{4 k ! \rho}\left(\frac{\sigma^{2}}{4}+r\right)^{k}\right) t^{k} \\
& =s-\frac{\sqrt{s s_{0}}}{\rho} \sum_{k=0}^{n} \frac{\left(\frac{\sigma^{2}}{8}+\frac{r}{2}\right)^{k} t^{k}}{k !}-\frac{s_{0}}{4 \rho} \sum_{k=0}^{n} \frac{\left(\frac{\sigma^{2}}{4}+r\right)^{k} t^{k}}{k !} \\
& =s-\frac{\sqrt{s s_{0}}}{\rho} \exp \left(\frac{\sigma^{2} t}{8}+\frac{r t}{2}\right)-\frac{s_{0}}{4 \rho} \exp \left(\frac{\sigma^{2} t}{4}+r t\right)
\end{aligned}
$$

The solution obtained above is in a complete agreement with the solution presented in the Theorem 3.0.2 [13].

To illustrate the result numerically, we make some choice for different values of $s$ and $t$, while treating all other parameter constant. Hence, for $r=0.06,|\rho|=0.01, \sigma=0.4$, and $S_{0}=4$. The plot of solution can be given as Figure 2. Now we compare the our solution $u_{R D T}$, i.e., equation (4.10), obtained by

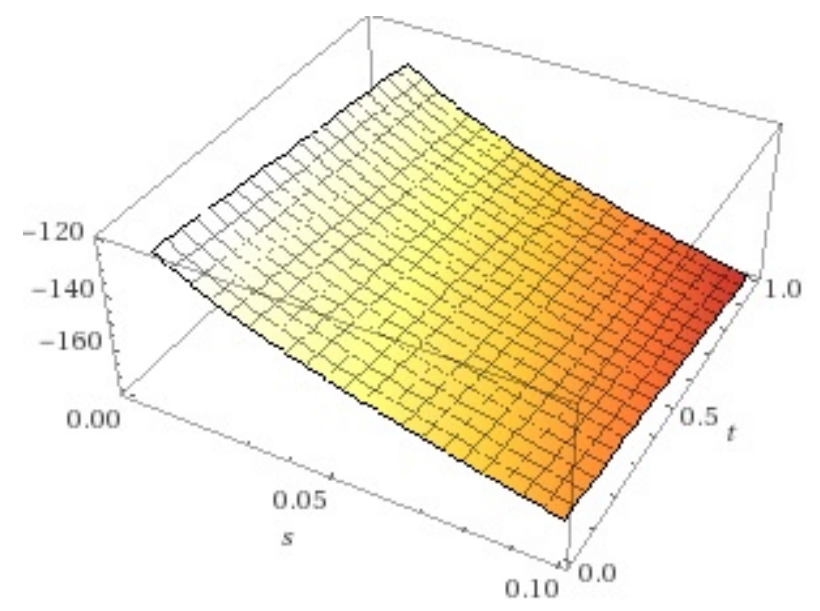

Figure 2

RDT algorithm with the solution $u_{A D M}$ obtained through adomian decomposition method in [20], by computing the relative error. For this we choose $s_{0}=4, r=0, \sigma=0.2, \rho=-0.01$. See Figures 3-5. 


$\begin{array}{cccc}s & u_{\mathrm{ADM}} & u_{\mathrm{RDTM}} & \left\|\frac{u_{\mathrm{RDTM}}-u_{\mathrm{ADM}}}{u_{\mathrm{ADM}}}\right\| \\ 0 & 100 & 100 & 0.00 \\ 0.5 & 241.921 & 248.992 & 0.0283985 \\ 1 & 301 & 311.000 & 0.0321543 \\ 1.5 & 346.449 & 358.696 & 0.0341431 \\ 2 & 384.843 & 398.985 & 0.0354449 \\ 2.5 & 434.539 & 418.728 & 0.0363857 \\ 3 & 466.731 & 449.41 & 0.0371113 \\ 3.5 & 496.374 & 477.666 & 0.0376893 \\ 4 & 524.000 & 504 & 0.0381679 \\ 4.5 & 549.977 & 528.764 & 0.0385707 \\ 5 & 574.574 & 552.214 & 0.0389158\end{array}$

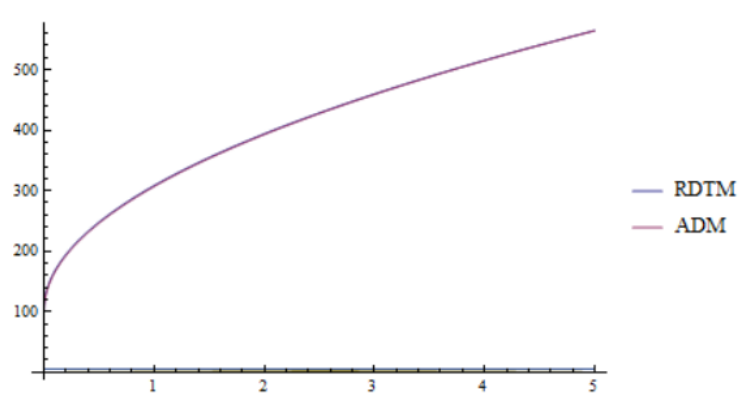

Figure 3: Comparison of $u_{R D T}$ and $u_{A D M}$ for $t=0.5$ years.
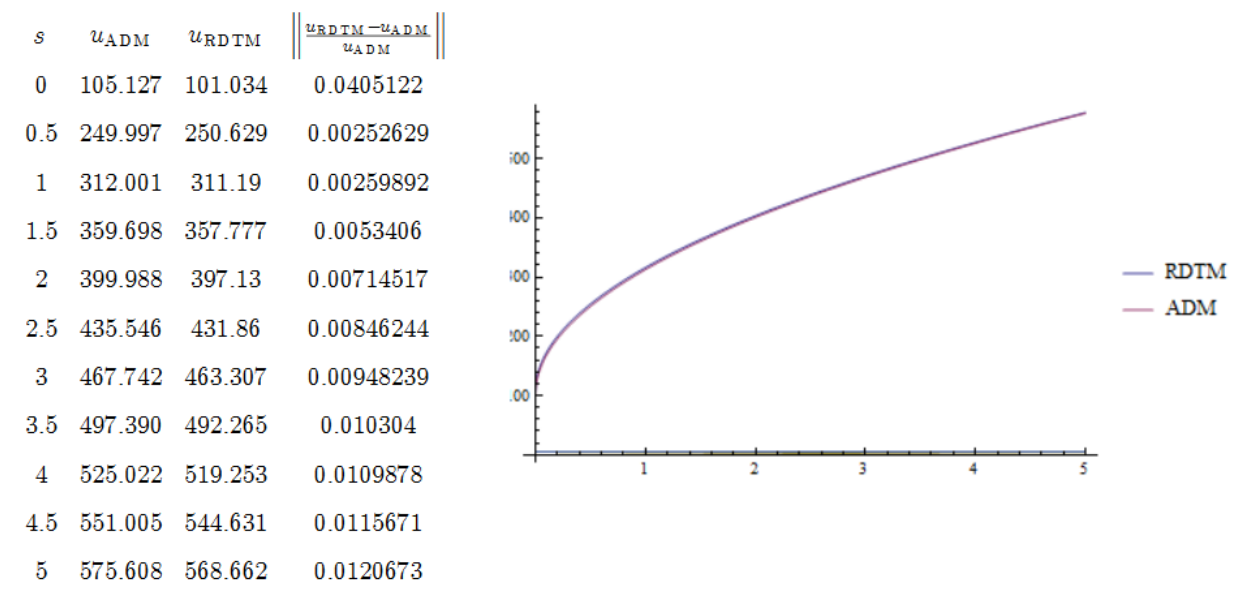

Figure 4: Comparison of $\mathfrak{u}_{R D T}$ and $\mathfrak{u}_{A D M}$ for $t=1$ years.

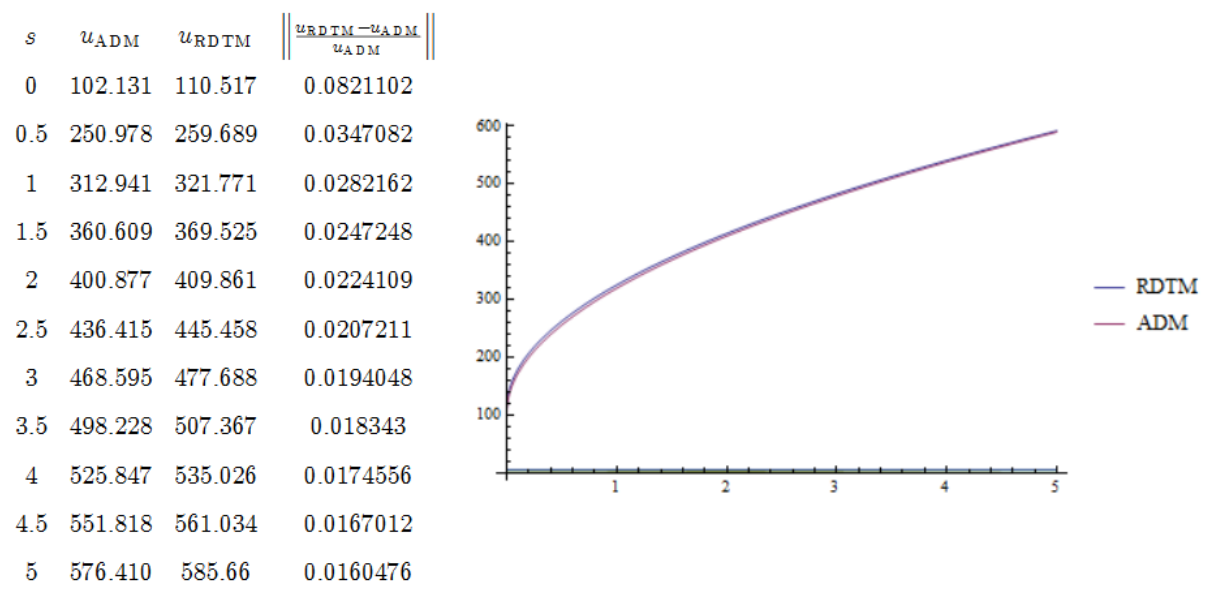

Figure 5: Comparison of $u_{R D T}$ and $u_{A D M}$ for $t=1.5$ years. 


\section{Pricing European option under relaxed Black-Scholes assumptions}

In this section, we aim to study two models proposed by Esekon in $[12,16]$ which govern the evolution of the price of the European option under Black-Scholes relaxed assumptions. The key relaxation was the inclusion of the transaction cost, which leads to the Black-Scholes market into illiquid and a nonlinear PDE. Let us begin by considering the following model in [16].

$$
\frac{\partial u}{\partial t}+\frac{1}{2} \sigma^{2} s^{2} \frac{\partial^{2} u}{\partial s^{2}}\left(1+2 \rho s \frac{\partial^{2} u}{\partial s^{2}}\right)=0,
$$

subject to initial condition

$$
u(s, 0)=\frac{1}{\rho}\left(-\sqrt{s} e^{\frac{\delta}{2}}+s(1-\ln s)\left(\frac{1}{4}-\frac{c}{\sigma^{2}}\right)-\frac{\sigma^{2}}{16 c} e^{\delta}\right) .
$$

Keep in view that the market-impact of hedging is directly proportional to the $\rho$. If $\rho \rightarrow 0$ or no hedging demand, the asset's price follows the standard Black-Scholes model with constant volatility.

For the solution of the equation (5.1), we aim to apply the reduced differential transformed algorithm. Using Definition 2.1 and properties of differential transform from Theorem 2.2 to equation (5.1), we arrive at the following recursive algorithm,

$$
(k+1) u_{k+1}=-\left[\frac{\sigma^{2} s^{2}}{2} \frac{\partial^{2} u_{k}}{\partial s^{2}}+\rho \sigma^{2} s^{3} A_{k}\right]
$$

where

$$
A_{k}=\sum_{k=0}^{h} \frac{\partial^{2} u(s, k)}{\partial s^{2}} \frac{\partial^{2} u(s, h-k)}{\partial s^{2}} .
$$

Let us now move towards the computation of the solution of the problem, (5.1)-(5.2).

When $k=0$ : By putting $k=0$ in the recurrence relation (5.3), we obtain

$$
u_{1}=-\left[\frac{\sigma^{2} s^{2}}{2} \frac{\partial^{2} u_{0}}{\partial s^{2}}+\rho \sigma^{2} s^{3} A_{0}\right]
$$

Now we compute $\frac{\partial^{2} u_{0}}{\partial s^{2}}$ and $A_{0}$ by using the initial condition (5.2), as follows,

$$
\begin{aligned}
\frac{\partial u_{0}}{\partial s} & =\frac{1}{\rho}\left[-\frac{1}{2 \sqrt{s}} e^{\frac{\delta}{2}}-\ln s\left(\frac{1}{4}-\frac{c}{\sigma^{2}}\right)\right] \\
\frac{\partial^{2} u_{0}}{\partial s^{2}} & =\frac{1}{\rho}\left[\frac{1}{4 s^{\frac{3}{2}}} e^{\frac{\delta}{2}}-\frac{1}{s}\left(\frac{1}{4}-\frac{c}{\sigma^{2}}\right)\right] \\
A_{0} & =\left(\frac{\partial^{2} u_{0}}{\partial s^{2}}\right)^{2}=\frac{1}{\rho^{2}}\left(\frac{e^{\delta}}{16 s^{3}}-\frac{e^{\frac{\delta}{2}}}{\left(\frac{1}{4}-\frac{c}{\sigma^{2}}\right)}+\frac{1}{s^{2}}\left(\frac{1}{4}-\frac{c}{\sigma^{2}}\right)^{2}\right) .
\end{aligned}
$$

Using the values of $\frac{\partial^{2} u_{0}}{\partial s^{2}}$ and $A_{0}$ in equation (5.4), we get

$$
\begin{aligned}
u_{1} & =-\frac{\sigma^{2} s^{2}}{2 \rho}\left(\frac{e^{\frac{\delta}{2}}}{4 s^{\frac{3}{2}}}-\frac{1}{s}\left(\frac{1}{4}-\frac{c}{\sigma^{2}}\right)\right)-\frac{\rho \sigma^{2} s^{3}}{\rho^{2}}\left(\frac{e^{\delta}}{16 s^{3}}-\frac{e^{\frac{\delta}{2}}}{2 s^{\frac{5}{2}}}\left(\frac{1}{4}-\frac{c}{\sigma^{2}}\right)+\frac{1}{s^{2}}\left(\frac{1}{4}-\frac{c}{\sigma^{2}}\right)^{2}\right), \\
& =\frac{1}{\rho}\left[-\sqrt{s} e^{\frac{\delta}{2}} \frac{c}{2}+\sigma^{2} s\left(\frac{1}{16}-\frac{c^{2}}{\sigma^{4}}\right)-\frac{\sigma^{2} e^{\delta}}{16}\right] .
\end{aligned}
$$


When $k=1$ : By putting $k=1$ in equation (5.3) we get,

$$
2 u_{2}=-\left[\frac{\sigma^{2} s^{2}}{2} \frac{\partial^{2} u_{1}}{\partial s^{2}}+\rho \sigma^{2} s^{3} A_{1}\right]
$$

Using (5.5) we have,

$$
\begin{aligned}
\frac{\partial u_{1}}{\partial s} & =\frac{1}{\rho}\left[-\frac{1}{2 \sqrt{s}} e^{\frac{\delta}{2}} \frac{c}{2}+\sigma^{2}\left(\frac{1}{16}-\frac{c^{2}}{\sigma^{4}}\right)\right], \\
\frac{\partial^{2} u_{1}}{\partial s^{2}} & =\frac{1}{\rho}\left[\frac{1}{4 s^{\frac{3}{2}}} e^{\frac{\delta}{2}} \frac{c}{2}\right], \\
A_{1} & =2 \frac{\partial^{2} u_{0}}{\partial s^{2}} \frac{\partial^{2} u_{1}}{\partial s^{2}}=\frac{2}{\rho^{2}}\left[\frac{c e^{\delta}}{32 s^{3}}-\frac{e^{\frac{\delta}{2}} c}{8 s^{\frac{5}{2}}}\left(\frac{1}{4}-\frac{c}{\sigma^{2}}\right)\right] .
\end{aligned}
$$

Putting the values of $\frac{\partial^{2} u_{1}}{\partial s^{2}}$ and $A_{1}$ in equation (5.6) we get,

$$
2 u_{2}=-\left[\frac{\sigma^{2} s^{2}}{16 \rho} \frac{e^{\frac{\delta}{2}} c}{s^{\frac{3}{2}}}+\rho \sigma^{2} s^{3} \cdot 2 \frac{1}{\rho^{2}}\left\{\frac{1}{16 s^{3}} e^{\delta} \frac{c}{2}-\frac{1}{4 s^{\frac{5}{2}}} e^{\frac{\delta}{2}} \frac{c}{2}\left(\frac{1}{4}-\frac{c}{\sigma^{2}}\right)\right\}\right] .
$$

Simplification yields,

$$
u_{2}=\frac{1}{\rho}\left(-\frac{1}{2} \sqrt{s} e^{\frac{\delta}{2}}\left(\frac{c}{2}\right)^{2}-\frac{\sigma^{2} e^{\delta} c}{32}\right) .
$$

When $k=2$ : For $k=2$ the equation (5.3) becomes,

$$
3 u_{3}=-\left[\frac{1}{2} \sigma^{2} s^{2} \frac{\partial^{2} u_{2}}{\partial s^{2}}+\rho \sigma^{2} s^{3} A_{2}\right] .
$$

Using (5.7) it follows that,

$$
\frac{\partial u_{2}}{\partial s}=-\frac{e^{\frac{\delta}{2}}}{4 \rho \sqrt{s}}\left(\frac{c}{2}\right)^{2}, \quad \frac{\partial^{2} u_{2}}{\partial s^{2}}=\frac{e^{\frac{\delta}{2}}}{8 \rho s^{\frac{3}{2}}}\left(\frac{c}{2}\right)^{2}
$$

Therefore,

$$
\begin{aligned}
A_{2} & =\frac{\partial^{2} u_{0}}{\partial s^{2}} \frac{\partial^{2} u_{2}}{\partial s^{2}}+\frac{\partial^{2} u_{1}}{\partial s^{2}} \frac{\partial^{2} u_{1}}{\partial s^{2}}+\frac{\partial^{2} u_{2}}{\partial s^{2}} \frac{\partial^{2} u_{0}}{\partial s^{2}} \\
& =\frac{1}{\rho}\left\{\frac{e^{\frac{\delta}{2}}}{4 s^{\frac{3}{2}}}-\frac{1}{s}\left(\frac{1}{4}-\frac{c}{\sigma^{2}}\right)\right\}\left\{\frac{1}{\rho} \frac{e^{\frac{\delta}{2}}}{8 s^{\frac{3}{2}}}\left(\frac{c}{2}\right)^{2}\right\}+\left\{\frac{1}{\rho} \frac{e^{\frac{\delta}{2}}}{4 s^{\frac{3}{2}}} \frac{c}{2}\right\}^{2}+\frac{1}{\rho}\left\{\frac{1}{8 s^{\frac{3}{2}}} e^{\frac{\delta}{2}}\left(\frac{c}{2}\right)^{2}\right\} \frac{1}{\rho}\left\{\frac{e^{\frac{\delta}{2}}}{4 s^{\frac{3}{2}}}-\frac{1}{s}\left(\frac{1}{4}-\frac{c}{\sigma^{2}}\right)\right\} \\
& =\frac{1}{\rho^{2}}\left[\frac{\sigma^{2} e^{\delta}}{8 s^{3}}\left(\frac{c}{2}\right)^{2}-\frac{\sigma^{2} e^{\frac{\delta}{2}}}{4 s^{\frac{5}{2}}}\left(\frac{c}{2 \sigma^{2}}\right)^{2}\left(\frac{1}{4}-\frac{c}{\sigma^{2}}\right)\right] .
\end{aligned}
$$

Using $\frac{\partial^{2} u_{2}}{\partial s^{2}}$ and $A_{2}$ into (5.7), we get

$$
u_{3}=\frac{1}{\rho}\left(-\frac{\sqrt{s} e^{\frac{\delta}{2}}}{4}\left(\frac{c}{2}\right)^{3}-\frac{\sigma^{2} e^{\delta} c^{2}}{64}\right) .
$$

Inductively it can be easily shown that

$$
u_{k}=\frac{1}{\rho}\left(-\frac{\sqrt{s} e^{\frac{\delta}{2}}}{4}\left(\frac{c}{2}\right)^{k}-\frac{\sigma^{2} e^{\delta}}{16}\left(\frac{c}{2}\right)^{k-1}\right), \text { for all } k \in \mathbb{N} .
$$


Hence we are in a position to obtain the solution of the problem (5.1)-(5.2),

$$
\begin{aligned}
u(s, t)= & \sum_{k=0}^{\infty} u_{k}(x) t^{k} \\
u(s, t)= & u_{0}(x)+u_{1}(x) t+u_{2}(x) t^{2}+u_{3}(x) t^{3}+\cdots \\
= & \frac{1}{\rho}\left(-\sqrt{s} e^{\frac{\delta}{2}}+s(1-\ln s)\left(\frac{1}{4}-\frac{c}{\sigma^{2}}\right)-\frac{\sigma^{2} e^{\delta}}{16 c}\right)+\frac{1}{\rho}\left(-\sqrt{s} e^{\frac{\delta}{2}} \frac{c}{2}+\sigma^{2} s\left(\frac{1}{16}-\frac{c^{2}}{\sigma^{4}}\right)-\frac{\sigma^{2} e^{\delta}}{16}\right) t \\
& +\frac{1}{\rho}\left(-\frac{1}{2} \sqrt{s} e^{\frac{\delta}{2}}\left(\frac{c}{2}\right)^{2}-\frac{1}{16} \cdot \frac{\sigma^{2} e^{\delta} c}{2}\right) t^{2}+\frac{1}{\rho}\left(-\frac{\sqrt{s} e^{\frac{\delta}{2}}}{4}\left(\frac{c}{2}\right)^{3}-\frac{\sigma^{2} e^{\delta} c^{2}}{64}\right) t^{3}+\cdots, \\
\rho u(s, t)= & -\sqrt{s} e^{\frac{\delta}{2}} \sum_{k=0}^{\infty}\left(\frac{c t}{2}\right)^{k}+s(1-\ln s)\left(\frac{1}{4}-\frac{c}{\sigma^{2}}\right)+\sigma^{2} s t\left(\frac{1}{16}-\frac{c^{2}}{\sigma^{4}}\right) \\
& -\frac{\sigma^{2}}{16 c} e^{\delta}\left(1+c t+\frac{1}{2}(c t)^{2}+\frac{1}{4}(c t)^{3}+\cdots\right), \\
u(s, t)= & \frac{1}{\rho}\left[-\sqrt{s} e^{\left(\frac{\delta+c t}{2}\right)}+s(1-\ln s)\left(\frac{1}{4}-\frac{c}{\sigma^{2}}\right)+\sigma^{2} s t\left(\frac{1}{16}-\frac{c^{2}}{\sigma^{4}}\right)-\frac{\sigma^{2}}{16 c} e^{(\delta+c t)}\right] .
\end{aligned}
$$

Thus we obtain the following solution which is in an exact agreement with the solution obtained by Esekon in Theorem 4.1 of [16],

$$
u(s, t)=\frac{1}{\rho}\left[-\sqrt{s} e^{\left(\frac{\delta+c t}{2}\right)}+s(1-\ln s)\left(\frac{1}{4}-\frac{c}{\sigma^{2}}\right)+\sigma^{2} s t\left(\frac{1}{16}-\frac{c^{2}}{\sigma^{4}}\right)-\frac{\sigma^{2}}{16 c} e^{(\delta+c t)}\right] .
$$

Let us now move towards the second model derived by Esekon in [12],

$$
\frac{\partial u}{\partial t}+\frac{1}{2} \sigma^{2} s^{2} \frac{\partial^{2} u}{\partial s^{2}}\left(1+2 \rho s \frac{\partial^{2} u}{\partial s^{2}}\right)=0
$$

subject to initial condition

$$
u(s, 0)=s-\frac{\sqrt{s s_{0}}}{\rho}-\frac{s_{0}}{4 \rho} .
$$

For the solution of the above equation (5.8), we apply the reduce differential transformed Algorithm to equation (5.8) and get the following,

$$
(k+1) u_{k+1}=-\left(\frac{\sigma^{2} s^{2}}{2} \frac{\partial^{2} u_{k}}{\partial s^{2}}+\rho \sigma^{2} s^{3} A_{k}\right)
$$

where

$$
A_{k}=\sum_{k=0}^{h} \frac{\partial^{2} u(s, k)}{\partial s^{2}} \frac{\partial^{2} u(s, h-k)}{\partial s^{2}} .
$$

Let us now move towards the computation of the problem (5.8)-(5.9).

When $k=0:$ We put $k=0$ in the recurrence relation (5.10) and obtain,

$$
u_{1}=-\left[\frac{\sigma^{2} s^{2}}{2} \frac{\partial^{2} u_{0}}{\partial s^{2}}+\rho \sigma^{2} s^{3} A_{0}\right]
$$

Now we compute $\frac{\partial^{2} u_{0}}{\partial s^{2}}$ and $A_{0}$, using initial condition (5.9),

$$
\frac{\partial u_{0}}{\partial s}=1-\frac{1}{2 \rho} \sqrt{\frac{s_{0}}{s}}, \quad \frac{\partial^{2} u_{0}}{\partial s^{2}}=\frac{1}{4 s \rho} \sqrt{\frac{s_{0}}{s}}, \quad A_{0}=\left(\frac{\partial^{2} u_{0}}{\partial s^{2}}\right)^{2}=\left(\frac{1}{4 s \rho} \sqrt{\frac{s_{0}}{s}}\right)^{2} .
$$


Using $\frac{\partial^{2} u_{0}}{\partial s^{2}}$ and $A_{0}$ in equation (5.11), we get

$$
u_{1}=-\left[\frac{\sigma^{2} s^{2}}{2}\left(\frac{1}{4 s \rho} \sqrt{\frac{s_{0}}{s}}\right)+\rho \sigma^{2} s^{3}\left(\frac{1}{4 s \rho} \sqrt{\frac{s_{0}}{s}}\right)^{2}\right]=-\frac{\sigma^{2}}{\rho}\left(\frac{\sqrt{s_{0} s}}{8}+\frac{s_{0}}{16}\right) .
$$

When $k=1$ : By putting $k=1$ in equations (5.3), we get

$$
2 u_{2}=-\left[\frac{\sigma^{2} s^{2}}{2} \frac{\partial^{2} u_{1}}{\partial s^{2}}+\rho \sigma^{2} s^{3} A_{1}\right]
$$

Now let us compute using (5.12),

$$
\frac{\partial u_{1}}{\partial s}=-\frac{\sigma^{2}}{16 \rho} \sqrt{\frac{s_{0}}{s}}, \quad \frac{\partial^{2} u_{1}}{\partial s^{2}}=\frac{\sigma^{2}}{32 s \rho} \sqrt{\frac{s_{0}}{s}}, \quad A_{1}=2 \frac{\partial^{2} u_{0}}{\partial s^{2}} \frac{\partial^{2} u_{1}}{\partial s^{2}}=\frac{\sigma^{2}}{64 s^{2} \rho^{2}} \frac{s_{0}}{s} .
$$

By using the values of $\frac{\partial^{2} u_{1}}{\partial s^{2}}$ and $A_{1}$ in equation (5.13) we get,

$$
2 u_{2}=-\left[\frac{1}{2} \sigma^{2} s^{2}\left(\frac{\sigma^{2}}{32 s \rho} \sqrt{\frac{s_{0}}{s}}\right)+\rho \sigma^{2} s^{3}\left(\frac{\sigma^{2}}{64 s^{2} \rho^{2}} \frac{s_{0}}{s}\right)\right]=-\frac{\sigma^{4}}{2 \rho}\left(\frac{\sqrt{s s_{0}}}{64}+\frac{s_{0}}{64}\right) .
$$

When $k=2$ : For $k=2$ the equation (5.3) becomes,

$$
3 u_{3}=-\left[\frac{1}{2} \sigma^{2} s^{2} \frac{\partial^{2} u_{2}}{\partial s^{2}}+\rho \sigma^{2} s^{3} A_{2}\right] .
$$

Using (5.14) it follows that,

$$
\begin{aligned}
\frac{\partial u_{2}}{\partial s} & =-\frac{1}{256 \rho} \sqrt{\frac{s_{0}}{s}} \sigma^{4}, \quad \frac{\partial^{2} u_{2}}{\partial s^{2}}=\frac{1}{512 s \rho} \sqrt{\frac{s_{0}}{s}} \sigma^{4} \\
A_{2} & =\frac{\partial^{2} u_{0}}{\partial s^{2}} \frac{\partial^{2} u_{2}}{\partial s^{2}}+\frac{\partial^{2} u_{1}}{\partial s^{2}} \frac{\partial^{2} u_{1}}{\partial s^{2}}+\frac{\partial^{2} u_{2}}{\partial s^{2}} \frac{\partial^{2} u_{0}}{\partial s^{2}}=\frac{\sigma^{4} s_{0}}{512 s^{3} \rho^{2}} .
\end{aligned}
$$

Substituting $\frac{\partial^{2} u_{2}}{\partial s^{2}}$ and $A_{2}$ into (5.15), we get

$$
3 u_{3}=-\left[\frac{\sigma^{2} s^{2}}{2}\left(\frac{1}{512 s \rho} \sqrt{\frac{s_{0}}{s}} \sigma^{4}\right)+\rho \sigma^{2} s^{3}\left(\frac{\sigma^{4} s_{0}}{512 s^{3} \rho^{2}}\right)\right]=-\frac{1}{6 \rho}\left(\frac{\sqrt{s_{0} s} \sigma^{6}}{512}+\frac{s_{0} \sigma^{6}}{256}\right) .
$$

We can see inductively that,

$$
u_{k}=-\frac{1}{\rho}\left(\frac{\left(\frac{\sigma^{2}}{8}\right)^{k}}{k !}+\frac{s_{0}}{4} \frac{\left(\frac{\sigma^{2}}{4}\right)^{k} t^{k}}{k !}\right), \text { for all } k \in \mathbb{N} .
$$

Hence we are in a position to explicitly write the exact solution of (5.8)-(5.9). Thus by the inverse RDT algorithm we get,

$$
\begin{aligned}
u(s, t) & =u_{0}(s)+\sum_{k=1}^{\infty} u_{k}(s) t^{k} \\
& =s-\frac{\sqrt{s s_{0}}}{\rho}-\frac{s_{0}}{4 \rho}-\frac{\sigma^{2}}{\rho}\left(\frac{\sqrt{s s_{0}}}{8}+\frac{s_{0}}{16}\right) t-\frac{\sigma^{4}}{2 \rho}\left(\frac{\sqrt{s s_{0}}}{64}+\frac{s_{0}}{64}\right) t^{2}-\frac{\sigma^{6}}{6 \rho}\left(\frac{\sqrt{s s_{0}}}{512}+\frac{s_{0}}{256}\right) t^{3}+\cdots, \\
& =s-\left(\frac{\sqrt{s s_{0}}}{\rho}+\frac{\sigma^{2}}{\rho} \frac{\sqrt{s s_{0}}}{8} t+\frac{\sigma^{4}}{2 \rho} \frac{\sqrt{s s_{0}}}{8} t^{2}+\frac{1}{6 \rho} \frac{\sqrt{s s_{0}} \sigma^{6}}{512}\right)-\left(\frac{s_{0}}{4 \rho}+\frac{\sigma^{2}}{\rho} \frac{s_{0}}{16}+\frac{\sigma^{4}}{2 \rho} \frac{s_{0}}{64} t^{2}+\frac{\sigma^{6}}{6 \rho} \frac{s_{0}}{256} t^{3} \ldots\right)
\end{aligned}
$$




$$
\begin{aligned}
& =s-\frac{\sqrt{s s_{0}}}{\rho} \sum_{k=0}^{\infty} \frac{\left(\frac{\sigma^{2}}{8}\right)^{k} t^{k}}{k !}-\frac{s_{0}}{4 \rho} \sum_{k=0}^{\infty} \frac{\left(\frac{\sigma^{2}}{4}\right)^{k} t^{k}}{k !}, \\
u(s, t) & =s-\frac{1}{\rho}\left(\sqrt{s s_{0}} \exp \left(\frac{\sigma^{2} t}{8}\right)+\frac{s_{0}}{4} \exp \left(\frac{\sigma^{2} t}{4}\right)\right) .
\end{aligned}
$$

The solution obtained above is in a complete agreement with the solution obtained in [12]. For numerical illustration, we will consider some examples for different values of $s$ and $t$, over fixed values for the other parameters. Hence, for $r=0.06,|\rho|=0.01, \sigma=0.4$, and $S_{0}=4$. The plot of solution can be given as Figure 6.

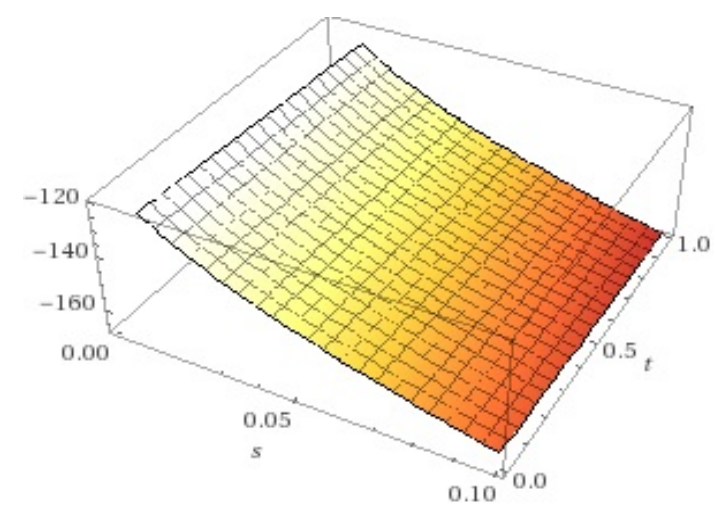

Figure 6

\section{Acknowledgments}

The author wish to express their gratitude to the anonymous referees and the editor for their valuable comments.

\section{References}

[1] T. Allahviranloo, S. S. Behzadi, The use of iterative methods for solving Black-Scholes equation, Int. J. Ind. Math., 5 (2013), 1-11. 1

[2] J. Ankudinova, M. Ehrhardt, On the numerical solution of nonlinear Black-Scholes equations, Comput. Math. Appl., 56 (2008), 799-812. 1

[3] D. Bakstein, S. Howison, A Non-Arbitrage Liquidity Model with Observable Parameters for Derivatives, Oxford Center for Industrial and Applied Mathematics (Working Paper), 2003 (2003), 52 pages. 1, 1, 3

[4] F. Black, M. Scholes, The pricing of options and corporate liabilities, J. Polit. Econ., 81 (1973), 637-654.

[5] M. Bohner, Y. Zheng, On analytical solutions of the Black-Scholes equation, Appl. Math. Lett., 22 (2009), 309-313. 1

[6] L. A. Bordag, A. Y. Chmakova, Explicit solutions for a nonlinear model of financial derivatives, Int. J. Theor. Appl. Finance, 10 (2007), 1-21. 1

[7] P. Boyle, T. Vorst, Option replication in discrete time with transaction costs, J. Finance, 47 (1992), 271-293.

[8] Z. Brzeźniak, J. Hussain, Global solution of nonlinear stochastic heat equation with solutions in a Hilbert manifold, Stoch. Dyn., 20 (2020), 29 pages. 1

[9] Z. D. Cen, A. Le, A robust and accurate finite difference method for a generalized Black-Scholes equation, J. Comput. Appl. Math., 235 (2011), 3728-3733. 1

[10] U. Çetin, R. A. Jarrow, P. Protter, Liquidity risk and arbitrage pricing theory, Finance Stoch., 8 (2004), $311-341$.

[11] R. Company, E. Navarro, J. R. Ramón Pintos, E. Ponsoda, Numerical solution of linear and nonlinear Black-Scholes option pricing equations, Comput. Math. Appl., 56 (2008), 813-821.

[12] J. E. Esekon, A particular solution of a nonlinear Black-Scholes partial differential equation, Int. J. Pure Appl. Math., 81 (2012), 715-721. 1 $5,5,5$

[13] J. E. Esekon, Analytic solution of a nonlinear Black-Scholes equation, Int. J. Pure Appl. Math., 82 (2013), 547-555. 4, 4 
[14] J. E. Esekon, Analytic solution of a nonlinear Black-Scholes equation with price slippage, Int. J. Pure Appl. Math., 96 (2014), 229-234. 3, 3

[15] J. E. Esekon, A Put option's value for a nonlinear Black-Scholes equation, Int. J. Pure Appl. Math., 105 (2015), $339-345$.

[16] J. E. Esekon, S. Onyango, N. Omolo-Ongati, Analytic solution of a nonlinear Black-Scholes partial differential equation, Int. J. Pure Appl. Math., 61 (2010), 219-230. 1, 5, 5

[17] R. Frey, Market illiquidity as a source of model risk in dynamic hedging, Risk Publications, London, (2000). 1

[18] R. Frey, P. Patie, Risk Management for Derivatives in Illiquid Markets: A Simulation Study, In: Advances in finance and stochastics, 2002 (2002), 137-159. 1

[19] R. Frey, A. Stremme, Market volatility and feedback effects from dynamic hedging, Math. Finance, 4 (1997), 351-374. 1, 1,1

[20] O. González-Gaxiola, J. Ruiz de Chávez, J. A. Santiago, Nonlinear Option Pricing Model Through the Adomian Decomposition Method, Int. J. Appl. Comput. Math., 2 (2016), 453-467. 4

[21] J. Hussain, On Existence and invariance of sphere, of solutions of constrained evolution equation, Int. J. Math. Comput. Sci., 15 (2020), 325-345. 1

[22] J. Hussain, B. Khan, On Cox-Ross-Rubinstein Pricing Formula for Pricing Compound Option, Int. J. Anal. Appl., 18 (2020), 129-148.

[23] J. Hussain, M. S. Khan, On the Pricing of Call-Put Parities of Asian Options by Reduced Differential Transform Algorithm, Int. J. Anal. Appl., 18 (2020), 513-530. 1

[24] L. Jódar, P. Sevilla-Peris, J. C. Cortés, R. Sala, A new direct method for solving the Black-Scholes equation, Appl. Math. Lett., 18 (2005), 29-32. 1

[25] Y. Keskin, G. Oturanç, Reduced Differential Transform Method for Partial Differential Equations, Int. J. Nonlinear Sci. Numer. Simul., 10 (2009), 741-749. 2.1, 2.2

[26] T. X. Li, N. Pintus, G. Viglialoro, Properties of solutions to porous medium problems with different sources and boundary conditions, Z. Angew. Math. Phys., 70 (2019), 18 pages. 1

[27] T. X. Li, G. Viglialoro, Analysis and explicit solvability of degenerate tensorial problems, Bound. Value Probl., 2018 (2018), 13 pages. 1

[28] H. Liu, J. M. Yong, Option pricing with an illiquid underlying asset market, J. Econom. Dynam. Control, 29 (2005), 2125-2156. 1

[29] S. R. Moosavi Noori, N. Taghizadeh, J. Manafian, Analytical approximations of one-dimensional hyperbolic equationwith non-local integral conditions by reduced differential transformmethod, Comput. Methods Differ. Equ., 8 (2020), 537-552. 2.3

[30] M. U. Rehman, J. Alzabut, J. Hussain, A. Hyder, On Spectral Properties of Doubly Stochastic Matrices, Symmetry, 12 (2020), 11 pages. 1

[31] M. R. Rodrigo, R. S. Mamon, An alternative approach to solving the Black-Scholes equation with time-varying parameters, Appl. Math. Lett., 19 (2006), 398-402. 1

[32] M. A. Soomro, J. Hussain, On study of generalized Novikov equation by Reduced differential transform method, Indian J. Sci. Tech., 12 (2019), 33-39. 1

[33] G. Viglialoro, T. E. Woolley, Boundedness in a parabolic-elliptic chemotaxis system with nonlinear diffusion and sensitivity and logistic source, Math. Methods Appl. Sci., 41 (2018), 1809-1824. 1 